\title{
Laser Photography — Examples of Processing of Image Information
}

\begin{abstract}
M. PISZCZEK*
Institute of Optoelectronics, Military University of Technology, S. Kaliskiego 2, 00-908 Warsaw, Poland

Modern cameras working in various spectral bands can be successfully applied in many areas of our lives. Observational capabilities of modern cameras are widely utilized in the area of public security systems while measurement functionalities of cameras are the domain of Machine Vision Systems used mainly in industry applications. The development of vision techniques and data processing methods changed the perception of vision systems as a multifunction, observation and measurement systems. Laser photography device, developed in the Institute of Optoelectronics, Military University of Technology is an example of such imaging device. One of properties of time-spatial framing method is the ability to perform complex spatial analysis of observed scene thanks to proper control of acquisition parameters and metadata processing. This property can be very useful especially in real-time spatial and geographic information systems. The paper presents theoretical applications of time-spatial framing method and first experimental results.
\end{abstract}

DOI: 10.12693 /APhysPolA.124.546

PACS: 42.30.Sy, 42.30.Tz, 06.30.Bp, 02.60.-x

\section{Introduction}

Dynamic development of imaging techniques leads to the growth of popularity of various cameras used in different areas of our lives. Image registration devices available on the market offer various functionalities which can be divided into two groups - observation and measurement functions.

Observation functionalities are mainly used in the public safety applications e.g. open area monitoring. Measurement functionalities are the domain of vision systems working mainly in industry and science applications.

Thanks to rapid development of data imaging and processing techniques, a camera can be regarded as an observation-measurement device. An example of such applications are area and satellite recognition (Fig. 1).
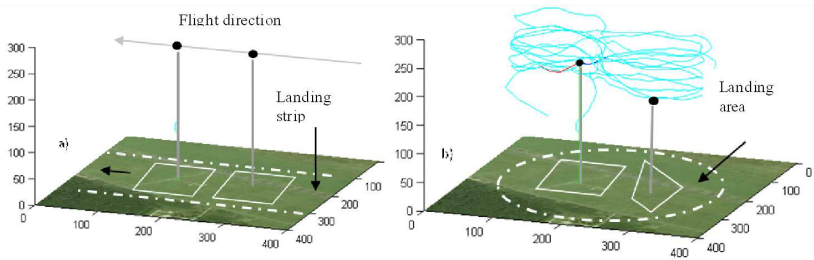

Fig. 1. Aero-photogrammetric terrain analysis (left satellite, right — area recognition).

A characteristic feature of this type of imaging information is the relatively fast acquisition of the spatial information. The quality and usability of the information can be described by two parameters: ground sampling distance (GSD) and ground resolved distance (GRD).

\footnotetext{
*e-mail: mpiszczek@wat.edu.pl
}

For fast estimation of information capabilities of imaging information, the ten degree scale - national imagery interpretability ratting scale (NIIRS) was introduced. This scale is the unique tool for objective image measurement and analysis.

From a point of view of imaging recognition, this system is a very valuable tool, but the interpretation of objects registered in the image visualized in vertical or oblique projection is not a simple task (humans are used to see the environment in the horizontal projection). If there was a solution acquiring visual information about objects in horizontal projection (including information of distance to objects) it would become a very valuable complement of all the tools used at the moment $[1,2]$.

A solution can be found amongst new imaging techniques. An example of a camera with observationmeasurement properties is the laser photography device (LPD) developed in the Institute of Optoelectronics, Military University of Technology. One of properties of the image acquisition method used in the LPD is the ability to perform spatial analysis with various complexity, by proper controlling of image acquisition parameters and metadata processing.

\section{Spatial framing}

Commonly used cameras have the property that they are able to define only the angular field of view of spatial imaging parameters. The values of this magnitude are obviously a derivative of the sensor size and the parameters of the optical system. The problem to evaluate the linear dimensions of objects can be solved with the knowledge of the distance to the object. In the case of air or satellite imaging, geometry of an object can be estimated by knowing the altitude of the aircraft. In the case of vision systems, one can use for example the information of the distance from the object to the camera on the 
production line. In many cases, however, you need additional information, not directly derived from the object's image registration device.

The classic photography does not provide the information about the distance to the observed objects. A time of flight ( $\mathrm{ToF}$ ) camera can be a solution. An example of a ToF camera is a laser photography device, which uses time-spatial framing method [3]. Thanks to this image acquisition method it is possible to precisely define the observation space (Fig. 2) by a few time and optics parameters.

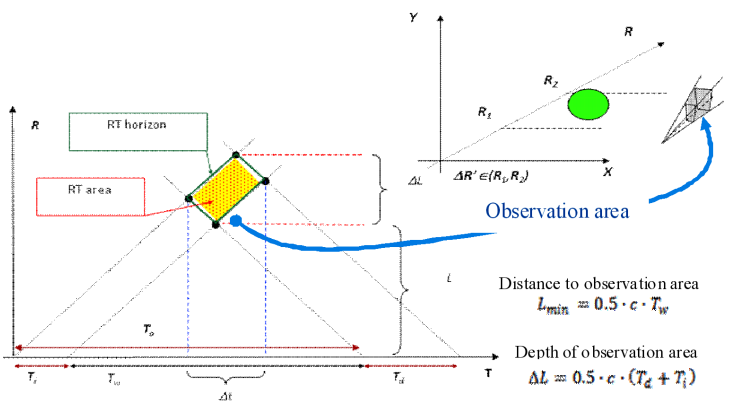

Fig. 2. Definition of observed space with the LPD.

Time parameters allow us to define minimal and maximum distance to scene registered in an image. Optic parameters are helpful to define width and height of image frame, thus objects presented in registered image can include only these objects which satisfy specific spatial conditions.

\section{Spatial analysis of vision information}

Air recognition systems or vision technical diagnostic systems assume the distance to observed object. In case of the LPD, the user is defining the distance to observed scene. Measurement capabilities of the device and image interpretation abilities depend not only on acquaintance of time parameters of acquisition but also on many other parameters. Only the result of analysis of various images with external data can guarantee the correctness of interpretation of scene registered in image frame. The experimental device registers simultaneously both images and metadata during image acquisition process. One can distinguish four groups of metadata: (a) time data; (b) spatial data; (c) radiometric data; (d) spectral data.

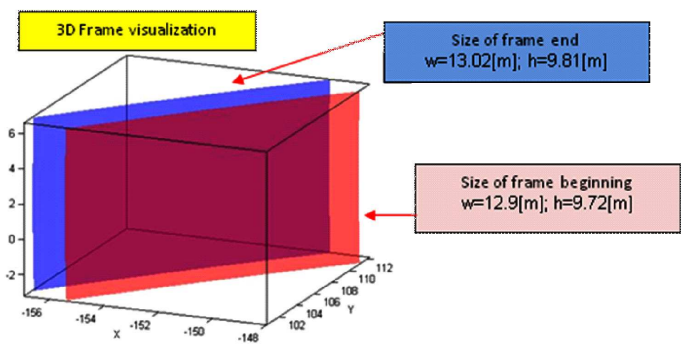

Fig. 3. Geometry of frame registered using the LPD.

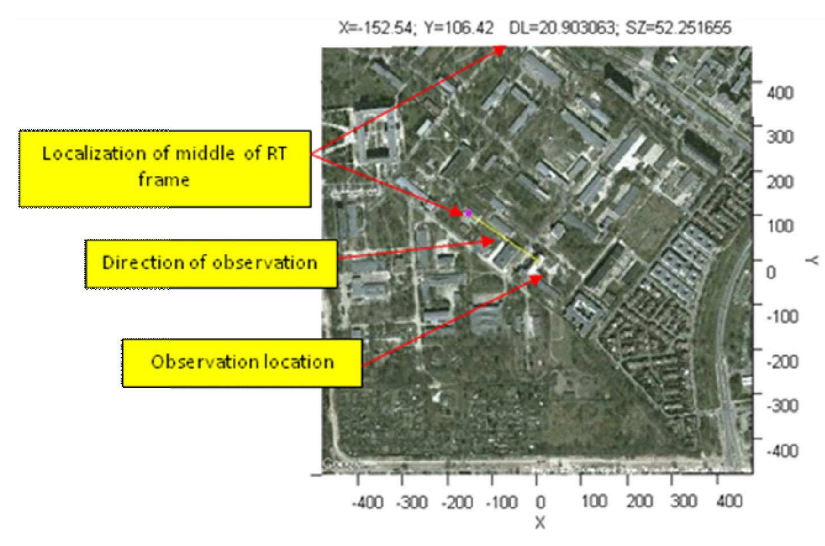

Fig. 4. Measurement scene positioning on map base.
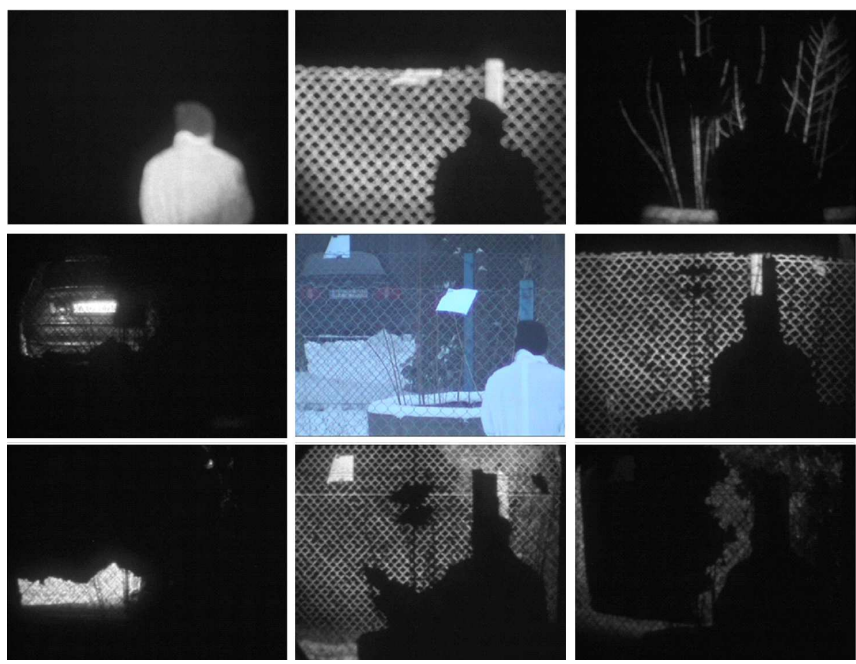

Fig. 5. Example of automatic scene segmentation by the LPD.

The key significance of acquisition of spatial information from images are mostly time and spatial data $[4,5]$.

Proper fusion of data and images make possible to achieve below functionalities: (a) frame geometry definition (scene fragment parameters registered on image - Fig. 3), (b) frame geolocalization in space (Fig. 4), (c) scene autosegmentation (Fig. 5).

\section{Tests}

Development of the experimental laser photography device allowed us to run many series of terrain tests. Test whose results are presented below was realized in the MUT area. The aim of the test was to scan the space (various distances with constant depth of observation) in selected direction.

Localization and direction of LPD observation are presented in Fig. 6.

Spatial parameters (distance and depth of observation) of test series are presented in Fig. 7. For analysis purposes, eight frames were selected.

Positioning of chosen frames on a base map are shown in Fig. 8. 


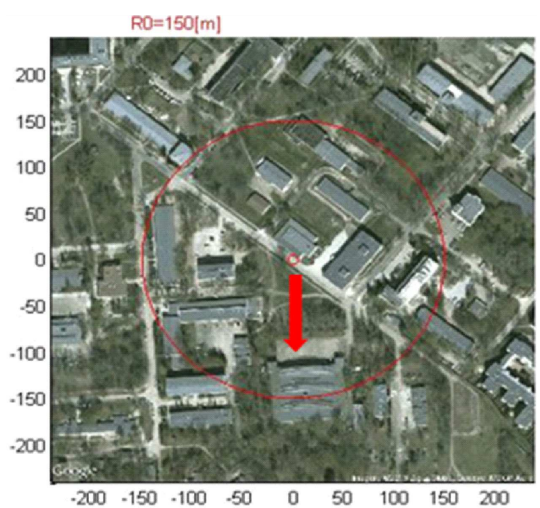

Fig. 6. Tests localization in MUT.

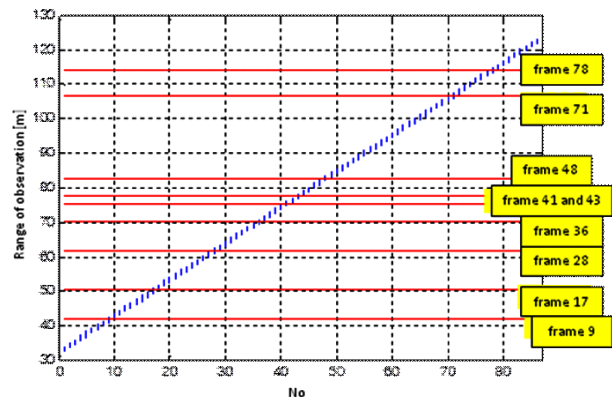

Fig. 7. Test series parameters.

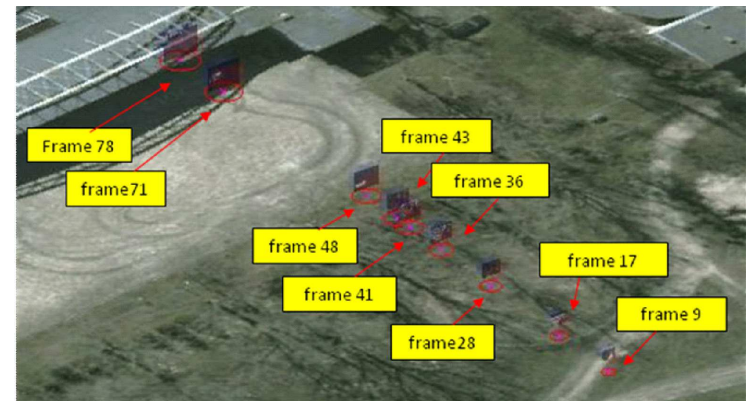

Fig. 8. Positioning of frames on a map.

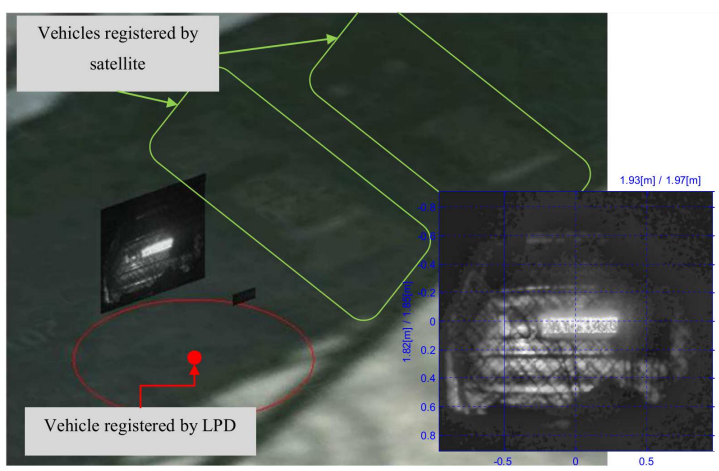

Fig. 9. Example of vehicle positioning.

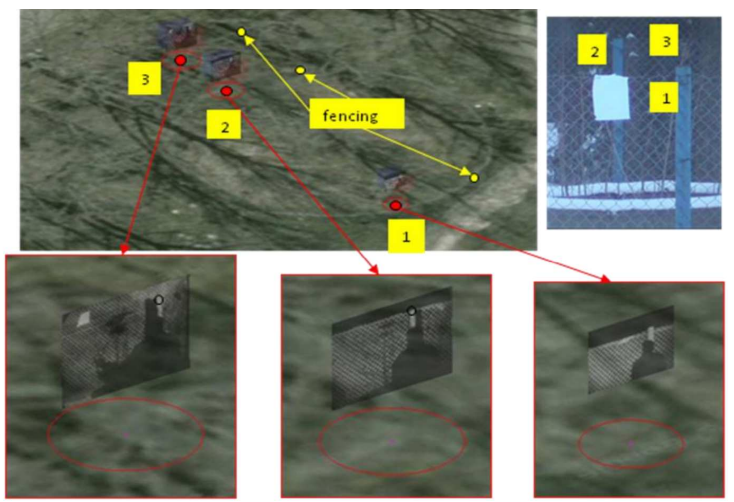

Fig. 10. Positioning of fencing elements.

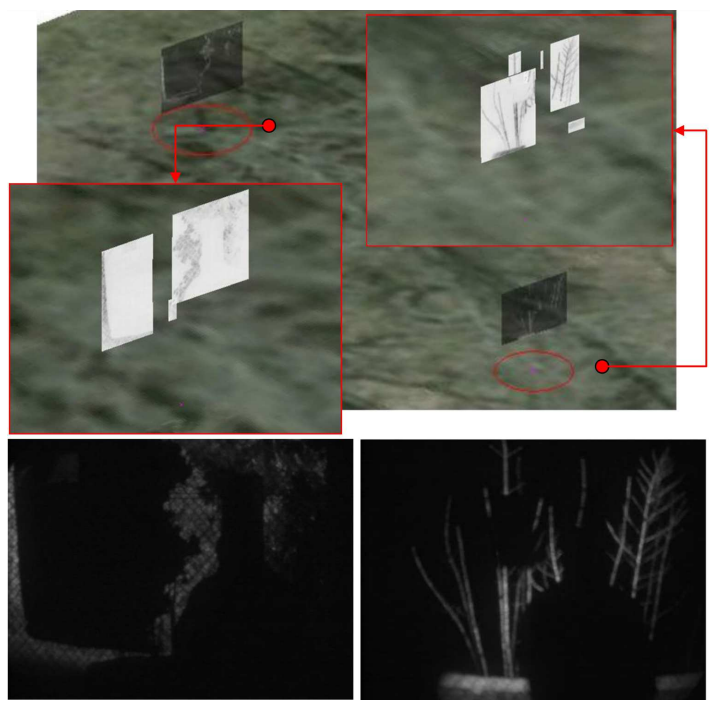

Fig. 11. Vision analysis of green flora identified based on satellite image.

Chosen elements of analysis are presented in the following figures:

(a) visualization of vehicle in the parking zone with metric net superimposed on the image (Fig. 9 - image shows vehicles registered on satellite image); (b) a precision of positioning frames on a map (Fig. 10 - elements of buildings - fencing), (c) visualization of trees and bush (Fig. 11).

\section{Summary}

Thanks to the laser photography, it is possible to lead observation not only of space on the distance defined by a user, but also to measure a geometry of registered objects. These properties of the laser photography device can be successfully applied in various systems of spatial and geographic information operating in real-time mode. Obviously, the area of operation of such information systems would be considerably smaller than of the air photogrammetry system (range would be limited to single $\mathrm{km}$ ). Fast update of information and easiness 
of interpretation are the undoubted advantages because of the horizontal visualization of terrain objects. These properties are especially useful in monitoring of open areas (critical management). Appropriate device/system software (which have been already tested) can be automatically updated with changing spatial situation. Visualization of imaging information can be realized using augmented reality techniques. Teams using such imaging information system can operate a tool offering significant possibilities of distributing information adapted to human perception.

\section{Acknowledgments}

This work was funded by the Polish Ministry of Science and Higher Education (project OR00000312).

\section{References}

[1] P. Andersson, Opt. Eng. 45, 034301 (2006).

[2] M. Brasebin, B. Bucher, C. Hoarau, Int. Arch. Photogram. Remote Sens. Spat. Inf. Sci. XXXVIII4/W15, 27 (2010).

[3] M. Piszczek, M. Kowalski, M. Szustakowski, K. Rutyna, K. Ludwikowski, Acta Phys. Pol. A 122, 862 (2012).

[4] M. Piszczek, Acta Phys. Pol. A 120, 716 (2011).

[5] M. Piszczek, M. Kowalski, Laser Photography in Selective Space Imaging and Navigation, Springer, Berlin 2013, Ch. IV. 